Revista de Psicología (UNLP)

https://revistas.unlp.edu.ar/revpsi

\title{
Intervenciones en dispositivos colectivos: ¿sobre qué transferencia? Reseña del ciclo internacional "autismo / transferencia / invención"
}

\author{
María Romé1,2 \\ Correspondencia \\ mrome@psico.unlp.edu.ar
}

\section{Filiaciones institucionales}

${ }^{1}$ Laboratorio de Investigaciones en Psicoanálisis $\quad{ }^{2}$ Consejo Nacional de Investigaciones Científicas y y Psicopatología (LIPPSI), Faculta de Psicología, Técnicas (CONICET, Argentina)

Universidad Nacional de La Plata (Argentina)

\section{Resumen}

En este manuscrito, se presenta la reseña del siguiente evento académico:

"Autismo / transferencia / invención". 4, 11 y 18 de noviembre de 2020. Ciudad Autónoma de Buenos Aires (modalidad virtual). Institución organizadora: La Cigarra (hospital de día para niñas, niños y adolescentes, perteneciente al Centro de Salud Mental y Acción Comunitaria Nº1, Ciudad Autónoma de Buenos Aires).

\section{Proceso editorial}

Recibido

3 mar. 2021

Aceptado

25 abr. 2021
1 ra decisión

28 mar. 2021

Publicado

26 abr. 2021
/ invención". Revista de Psicología,

20(2), 217-224. HTTPs://DX.DOI.

ORG/10.24215/2422572XE092
ISSN

2422-572X

Licencia

Licencia de Cultura Libre CC-BY 4.0

(Compartir - Adaptar - Atribuir)

Entidad editora

RevPsi es una publicación de la

Facultad de Psicología (Universidad

Nacional de La Plata, Argentina) 
En noviembre del 2020 se realizó, de manera virtual, el ciclo internacional titulado "Autismo / transferencia / invención", organizado por La Cigarra, hospital de día para niñas, niños y adolescentes perteneciente al Centro de Salud Mental y Acción Comunitaria №1 (CABA). Además, participaron en este ciclo las siguientes instituciones invitadas: Associació TEAdir (Barcelona, España), Hacer Lugar (CABA, Argentina), Fundación Avenir (Córdoba, Argentina), Antenne 110 (Bruselas, Bélgica), escuelas infantiles Patinete y Espacio Torreón (Zaragoza, España).

La presente reseña trata de seguir el hilo del tema central de este ciclo: la cuestión de la transferencia en la práctica del psicoanálisis aplicado a la institución, más específicamente, en dispositivos colectivos de orientación lacaniana ideados para el abordaje de psicosis, autismos y otras presentaciones actuales de la infancia y de la adolescencia.

\section{Lógica de los dispositivos colectivos y modalizaciones de la transferencia}

Ya en la introducción al ciclo, Gustavo Slatopolsky -coordinador de la cigarrapropone una primera idea de la transferencia en la práctica institucional: punto de encuentro entre un arreglo advertido, del lado del dispositivo, y el arreglo singular con el que llega cada uno. Se tratará entonces a lo largo de este evento de analizar la invención que propone cada institución para alojar la propia de cada sujeto. Se destaca que la discusión sobre la transferencia implica una conversación sobre la lógica de los dispositivos. Esto permitirá, según Erick González Guzmán -TEAdir-, hacer una lectura de la institución en sí misma, preguntarnos en qué consiste y en qué se sostiene la práctica del psicoanálisis allí.

Desde las diferentes instituciones, se coincide en plantear que hay algo del orden de la transferencia que sostiene el trabajo en los dispositivos colectivos propuestos. No obstante, comparten también la pregunta: ¿De qué transferencia se trata? Junto con este, surge el interrogante acerca de si es posible la transferencia analítica en la institución. Es decir, si hay allí analista y, por lo tanto, opera algo del orden del acto analítico. O bien, si es posible la transferencia sin analista.

Tales interrogantes se entraman con otra cuestión problemática, que es la de la transferencia en el autismo. Una referencia mencionada en la exposición de González Guzmán anuda ambas cuestiones. En vez del operador del sujeto supuesto saber, que produciría el equívoco que funciona como resorte de un análisis, tendríamos en cambio al "sujeto supuesto interesarse" (Briole, 2000, p.6): lugar a ocupar por el interviniente, a partir de su interés por el trabajo que realiza el sujeto en el autismo. El índice de este operador no es el significante de la transferencia, sino el "instante de atención" del sujeto autista (Laurent, 2013, p.125).

Para poner a prueba la operatividad de esa noción, González Guzmán presenta un caso que muestra cómo en el trabajo con un sujeto llamado autista se alcanza ese instante de atención, abriendo entonces un cierto juego de la transferencia. Al retomar una 
observación de Maleval sobre el tratamiento de Joey, "el niño-máquina” (Maleval, 2011, p.41), llevado adelante por Bettelheim (1967/1983), se destaca la importancia de preservar la máquina con la que llega el sujeto, hasta el final de su itinerario. Ahora bien, considerando que no se trata de preservarla inmutable, la pregunta que circula, tanto en este como en otros casos presentados a lo largo del ciclo, es la referida a cuándo intervenir.

Como una de las modalidades de la transferencia se menciona la propuesta de Stevens, quien plantea que la entrada de un sujeto en la institución es posible cuando esta consigue introducirse en su síntoma (Stevens y Hellebois, 2006, en Ruiz, 2019). Del mismo modo, Lacan (1964-1965/2006) se refería al psicoanalista como complemento del síntoma. González Guzmán sostiene entonces que una institución debe insertarse en el síntoma del sujeto de manera tal que su modo de goce quede alterado definitivamente y que, para obtener el goce preciso para sostenerse, necesite recurrir a la institución.

En su comentario al trabajo de TEAdir, Ricardo Seijas -la cigarra- retoma el problema de la transferencia en el autismo: el lazo con el autista, ¿puede llamarse transferencia analítica? ¿Hay allí acto analítico? Nos recuerda entonces que esta cuestión sólo puede responderse desde el a posteriori, a partir de los efectos que provoca una intervención en la posición del autista, en su enunciación. Si bien es sabido, a partir de Maleval (2011), que la posición del autista es de rechazo a la enunciación, Seijas plantea que lo que encontramos en la clínica es que dentro de esa posición hay variaciones, que se producen y se leen en transferencia.

En el caso presentado se subraya una cierta tensión que aparece en la dirección de la cura. Inicialmente sostenida en la idea de lateralizar la transferencia, es decir, multiplicarla con respecto a diversos partenaires, la posición del sujeto es la de insistir en elegir a uno o dos de estos, con los cuales constituye un lazo completamente distinto a otros. Esa modalidad de la transferencia genera en él una fuerte dependencia con respecto a cierto interviniente. Si bien al principio fueron dóciles a esa transferencia adhesiva, esto no fue sin cierta incomodidad. Tal adhesividad resultaba funcional a la inercia de su defensa.

Con respecto a dicha tensión en la dirección de la cura, Seijas propone diferenciar la transferencia adhesiva del lazo privilegiado con un analista, con un Uno, que hizo posible la eficacia de ciertas intervenciones. La pregunta que surge entonces es si podemos nombrar a tales intervenciones como acto del analista, teniendo en cuenta que, como efecto, el sujeto cambia de posición en relación con su decir, alcanzando finalmente un lugar de enunciación inédito.

Ahora bien, según González Guzmán, la dirección de algunas intervenciones en el caso presentado apunta a la destitución de ciertos Unos, en el momento en que estaban dadas las condiciones para la práctica entre varios. El gran interrogante que se plantean en TEAdir es cómo sostener una transferencia sin analista, en tanto los operadores funcionan como "intervinientes", aunque no sin una cierta transferencia al psicoanálisis, al campo freudiano. La pregunta es entonces si es posible pensar 
la transferencia sin analista. A partir de ese interrogante se esbozan posiciones divergentes, que dan lugar a un debate sostenido a lo largo del ciclo.

Por un lado, encontramos la posición de Claudia Lijtinstens -Fundación Avenir-, quien plantea que la práctica en institución debe leerse como una de las "variantes de la cura-tipo" (Lacan, 1955/2003, p. 311): no hay transferencia, interpretación ni cura en el sentido estricto de estos términos; no hay analista ni acto del analista, sino a lo sumo acciones que pueden devenir en intervención. No obstante, plantea que jugar la partida cada vez implica recrear ese partenaire necesario, hacer que el sujeto lo requiera en su invención. Como dimensión posible de la transferencia en la institución, Lijtinstens destaca la "transferencia de trabajo": aquella que se sostiene de un fuerte lazo de los miembros del equipo con la escuela u orientación. Entonces, ¿cómo puede haber transferencia sin analista? Según Lijtinstens no hay analista pero hay reunión de equipo, que es de anudamiento clínico. Allí la transferencia está anudada por una orientación, en la cual los miembros del equipo operan en tanto "analizantes civilizados”, expresión de Laurent recuperada por Stevens (2004/2020, p. 8).

Por otro lado, encontramos la posición de Slatopolsky, en representación de la cigarra, que sostiene que es posible la transferencia sin analista, pero habría que ver de qué transferencia se trata. ¿No sería transferencia salvaje? Es decir, un lazo transferencial sin una lectura analítica. Surge entonces la pregunta por el valor o la eficacia de esa modalidad transferencial. ¿Qué incidencia clínica puede tener esa transferencia, sin una lectura a posteriori de sus efectos?

De allí se desprende otro interrogante, que surge del trabajo de TEAdir. Al considerar la transferencia privilegiada del sujeto en cuestión con respecto a una interviniente, la pregunta es si hay que ir en contra de esa transferencia, es decir lateralizarla, o bien se trata de soportarla, para poder en un segundo tiempo transformarla, agujerear ese modo de lazo. Más allá de esta cuestión, las diferentes instituciones coinciden en que es preciso hacer una lectura de la transferencia, entendida como una solución del sujeto de la cual el analista forma parte.

En articulación con lo anterior, desde La Cigarra sostienen que en la práctica colectiva no sólo es posible sino que es preciso un acto analítico para que haya del analista en la institución, como una presencia evanescente que es leída de ese modo desde el après-coup, en función de los efectos que provoca. Ese acto sería solidario de algo del orden de la transferencia a un Uno, lazo transferencial privilegiado del sujeto con un interviniente, que se distingue de los demás por los efectos que produce en él.

Por su parte, Gracia Viscasillas -escuelas infantiles Patinete y Espacio Torreónpropone una distinción, a partir de su experiencia en la práctica del psicoanálisis en institución: diferenciar la transferencia como efecto de la transferencia como producto o producción. La primera, transferencia como efecto, requiere de cada institución inventar una modalidad de acción que le sea conforme al niño autista, modalidad de acción que puede tomar el nombre de práctica entre varios, en sus diversas formas. Viscasillas plantea que hay ciertas condiciones previas en el modo de trabajo de cada institución, que están ahí antes de la llegada de un 
niño, que implican la oferta de un Otro pluralizado, no invasivo, un lugar fuera de exigencia; unos otros que alojarán sus modos de estar, que estarán atentos al detalle e intentarán asociarse a su hacer buscando siempre el consentimiento del sujeto. Esa modalidad de acción crea una cierta "atmósfera" de la institución (Di Ciaccia, 2005) que hace el lugar vivible para el niño.

Ahora bien, más allá de esas condiciones previas, dicha atmósfera puede crear efectos transferenciales, que son sancionados como tales a partir del consentimiento del niño. Tales efectos consisten en cierta imbricación entre la invención de la institución y la del niño, que puede entonces hacerse más permeable y dar lugar al lazo con otros.

La transferencia como producto sería en cambio la producción de un nuevo lazo transferencial que surge como acontecimiento en el trabajo con un niño; tal acontecimiento determina un origen, separa un antes y un después. Opera de ese modo tanto para el niño como también para el interviniente, que puede ocupar a partir de ahí un lugar de partenaire. Es esta modalidad transferencial la que hace acto, marcando una discontinuidad en la posición del sujeto y en su relación con el Otro. En ese punto, podríamos decir que la posición de Viscasillas se acerca a la de La Cigarra con respecto a la posibilidad del acto analítico en la práctica institucional.

A continuación, Marita Manzotti presenta "Hacer Lugar" -de la que es fundadoracomo una institución cuya propuesta es sostener un tratamiento psicoanalítico individual, a partir de un dispositivo novedoso al que llaman "dispositivo soporte". Se trata de una oferta caso por caso, en la que sólo participan practicantes del psicoanálisis. Agrega que tal posición de anfitrión puede alojar a maestros, a profesionales de otras disciplinas y a familiares, para mantener con ellos una lectura de las particularidades de las situaciones que se presentan en el devenir del trabajo. La define entonces como una institución que arma red pero por fuera, soporte para cada niño.

Ahora bien, más allá de la presencia exclusiva del psicoanálisis en la institución, aclara que en ella no hay analistas sino analizantes decididos, practicantes del psicoanálisis que sostienen una operatoria de descompletamiento a la que denominan el "más que Uno". Desde el armado mismo del dispositivo, su lógica introduce la dimensión equívoca, la falta, el no-todo, que no sin cálculo se despliega en cada encuentro. El dispositivo soporte, que crearon para la práctica en esta institución, tiene por objetivo sostener, habilitar que el trabajo del parlêtre no se trabe ni se obstaculice con la presencia de los practicantes, pero sí se amplíe a partir de la puesta a prueba con alguna diferencia o equívoco. Se trata entonces de tomar sólo su producción y desde allí operar con mínimos tropiezos, atentos a sus respuestas.

Mediante la lógica del "aserto de certidumbre anticipada" (Lacan, 1966/2003, p.187), en esta institución proponen ordenar el trabajo clínico en tres tiempos y dos escansiones; tiempos no cronológicos y por tanto de duración variable. El tercer tiempo, momento de concluir, es aquel en que Lacan (1966/2003) ubica la prisa por concluir en un acto, en el que cobra valor todo el dispositivo. Para los intervinientes es 
el momento de una espera anticipada en un cruce de camino inesperado para el niño, un punto de encuentro que si se produce introduce una verificación en acto. De esta manera, podríamos decir que la posición de Hacer Lugar en el debate con respecto a la cuestión del acto del analista es la de considerar que es posible en institución, alineándose entonces con lo planteado por La Cigarra y los dispositivos de Zaragoza.

Con respecto a la cuestión de la transferencia, Manzotti subraya la importancia de la relación transferencial de los padres o familias con la institución, como condición previa al trabajo con el niño. Se trata entonces de un dispositivo que oferta un trabajo individual, pero con una fuerte inclusión de las familias, así como de las escuelas.

En el comentario a esta presentación de Manzotti, a cargo de Marta Gutiérrez y Howard Rouse -TEAdir-, se plantean algunos interrogantes que quedan abiertos. La propuesta de Hacer Lugar es la de sostener un tratamiento individual, pero el resto de la presentación parece ir a contrapelo de esa propuesta. El "más que Uno" posibilita un tratamiento del Otro, apunta a descompletar al Otro. La pregunta es entonces si el dispositivo soporte se inscribe en la línea de la práctica entre varios.

Considerando que las dos últimas presentaciones evocadas -la de Hacer Lugar y la de las instituciones de Zaragoza- recortan intervenciones en las cuales el sujeto se encuentra de alguna manera a solas con quien ocupa el lugar del analista, surge la pregunta acerca del papel de lo colectivo en la institución. Interrogante que podría hacerse extensivo a las demás instituciones presentadas.

Como vemos, a lo largo del ciclo insiste la cuestión de cómo anudar transferencia y dispositivo colectivo. A partir de las conversaciones y discusiones desplegadas, empiezan a tomar forma distintas modalidades de la transferencia en institución, que podrían agruparse retomando algunos puntos ordenados por Martina Cicchetti y Ricardo Seijas en sus presentaciones. En primer lugar, encontramos la transferencia al psicoanálisis, es decir, los lazos de los intervinientes al psicoanálisis como saber y a cierta orientación de la clínica. Se trata de un saber fallido, agujereado estructuralmente: falla que en cada dispositivo se trabaja y se sostiene en los espacios de elaboración colectiva. Esto se expresa en el lugar fundamental que adopta en todas las instituciones la transferencia a la reunión de equipo como espacio de elaboración de la experiencia, articulación teórica y formalización de la práctica.

En segundo lugar, es posible recortar la transferencia al saber hacer del parlêtre: el hecho de alojar a un sujeto con su síntoma en la institución de alguna manera invierte la cuestión de la transferencia; no hay sujeto supuesto saber, pero será preciso aprender del sujeto y de cómo se las arregla, gracias a su saber hacer con el goce. Es él a quien se supone saber arreglárselas con su propio goce, con el ruido de la lengua, que será preciso leer y respetar.

En tercer lugar, podemos mencionar la transferencia de los padres/ las familias con respecto a la propuesta de la institución como condición previa al tratamiento: dimensión transferencial mencionada por las distintas instituciones, surgiendo algunas preguntas al respecto, como la de Pedro Gras -Espacio Torreón- a Slatopolsky, con 
relación al movimiento en el lugar de los padres en el dispositivo a partir del trabajo en la web: ¿cuál era antes el lugar de los padres, en la presencialidad? ¿Cuál es ahora?

En cuarto lugar, se destaca la importancia de la transferencia de los participantes al dispositivo, también llamada "transferencia a la máquina" en la cigarra. En esta dimensión, la dirección de la cura en la práctica entre varios apunta a lateralizar la transferencia, a producir una transferencia múltiple o plural, dirección que de alguna manera se interroga, se pone a prueba en la presentación de TEAdir.

Tal modalidad transferencial puede adoptar un estatuto particular cuando el dispositivo pasa a formar parte del síntoma, la solución o la defensa del parlêtre. Seijas propone hablar de transferencia al dispositivo cuando esto sucede y aclara que ese lazo no se constituye sólo porque el dispositivo se acomode a las defensas del sujeto, sino también porque le va ofreciendo pequeñas diferencias. En sus diversas formas, tales diferencias apuntan a incomodar la defensa: parten de cierto respeto por el saber hacer con el goce, pero al mismo tiempo implican un deseo de otra cosa, una espera al sujeto en otro lugar.

Por último, en el transcurso del ciclo se hizo referencia a la transferencia al Uno: solidaria del acto del analista, esta modalidad transferencial marca un antes y un después en las coordenadas de goce del parlêtre posibilitando una nueva escritura, una nueva defensa, un nuevo síntoma. Se trata entonces de un lazo privilegiado del sujeto a un Uno de la institución, lugar del analista en el dispositivo que Seijas propone denominar sinthome-agujero.

\section{A modo de conclusión}

A lo largo de este ciclo se presentaron diversos dispositivos institucionales ideados para el abordaje de psicosis, autismos y otras presentaciones actuales del malestar en la infancia y adolescencia. Si bien tales dispositivos presentan configuraciones de lo más diversas, comparten una misma ética y convergen en una misma orientación: la orientación por el síntoma del sujeto, concebido desde la perspectiva del psicoanálisis en sus dos caras, como sufrimiento o malestar y como arreglo o solución. En consonancia con esa orientación, en las diferentes instituciones presentadas se preguntan cómo alojar el síntoma del sujeto y cómo incidir en él. Más allá de los matices señalados al respecto, coinciden en plantear que hay algo del orden de la transferencia que sostiene la práctica en los dispositivos colectivos propuestos: transferencia de trabajo, transferencia al saber hacer del parlêtre, transferencia a la institución, a la máquina, al analista en tanto Uno. Asimismo, coinciden en sostener la importancia de la lectura desde el a posteriori de tales modalidades transferenciales, que motorizan y sostienen el trabajo del psicoanálisis en la institución. 


\section{Referencias}

Bettelheim, B. (1967/1983). La forteresse vide. L'autisme infantile et la naissance de soi. Gallimard.

Briole, G. (2000). À bâtons rompus. L'Essai, 3, 3-9.

Di Ciaccia, A. (2005). La pratique à plusieurs. La Cause Freudienne, 61, 107-118.

Lacan, J. (1955/2003). Variantes de la cura-tipo. En sus Escritos I (T. Segovia, trad.) (pp. 311-348). Siglo XXI.

Lacan, J. (1964-1965/2006). El seminario, libro XII, Problemas cruciales para el psicoanálisis ( $\mathrm{R}$. Rodríguez Ponte, trad.). Escuela Freudiana de Buenos Aires.
Lacan, J. (1966/2003). El tiempo lógico y el aserto de certidumbre anticipada. Un nuevo sofisma. En sus Escritos I (T. Segovia, trad.) (pp. 187-203). Siglo XXI.

Laurent, É. (2013). La batalla del autismo: de la clínica a la política. Grama.

Maleval, J.-C. (2011). El autista y su voz. Gredos.

Ruiz, I. (2019). Interrogar el entre varios. L'Atelier, $1,68-74$.

Stevens, A. (2004/2020). La institución: práctica del acto. L'Atelier, 3, 11-21. 